\title{
DER FUR PFERDE VERSCHIEDENEN GEWICHTS UND ALTERS ANZUSETZENDE ZUGWIDERSTAND
}

\author{
Mikko Varo und Viljo Vainikainen \\ Zentrale für Land: irtschaftliche Forschung, Abteilung fïr Haustierziichtung, \\ Tikkurila
}

Eingegangen am 28. III. 1958

Bei der Züchtungsarbeit, in der man es auf das Entwickeln der Zugkraft des finnischen Pferdes abgesehen hat, ist die Zugkraft der Hengste von dem Jahre 1936 an erst durch den von Professor T. TERHo konstruierten mittels eines Federmechanismus funktionierenden Widerstandmessers sowie später durch einen von Techniker E. Elmo gebauten hydraulischen Messer in einem von Vainikainen (4) näher beschriebenen Verfahren gemessen worden. Eines der wichtigsten Ziele beim Messen der Zugkraft hat darin bestanden, die in bezug auf das Gewicht relative Zugkraft herauszustellen, damit man bei der Auslese nicht unbegründet Pferde stattlicher Grösse bevorzuge, da deren kilogrammässige Zugleistung naturgemäss im allgemeinen grösser als die der kleinen Tiere ausfällt. Die in bezug auf das Gewicht relative Zugkraft hat man auf die Weise herausgestellt, dass der Betrag des Zugwiderstandes bei jeder $50 \mathrm{~m}$ langen Stufe des Zugversuchs als ein in bestimmter Grösse anzugebender prozentualer Anteil am Lebendgewicht des Pfredes festgelegt worden ist. Der Zugwiderstand der ersten Stufe hat bei vierjährigen Pferden $25 \%$ und seine Steigerung bei jeder folgenden Stufe $5 \%$ vom Lebendgewicht ausgemacht. Um 12 Stufen zu erreichen, hat also ein $500 \mathrm{~kg}$ schweres Pferd eine Last mit einer Kraft von $400 \mathrm{~kg}$ und ein $700 \mathrm{~kg}$ schweres mit einer Kraft von $560 \mathrm{~kg}$ zu ziehen.

Da der Zugversuch bei den Gelegenheiten des Eintragens in das Stammbuch der Pferde als einziger die Zugkraft des Tieres messender Versuch ausgeführt wird, müsste er recht hohen Ansprüchen genügen. Nicht nur dass die Versuchsergebnisse selbst bei ihrer Erlangung unter verschiedenen Verhältnissen vergleichbar sein sollten, sie hätten auch insofern gerecht zu sein, als das Versuchsverfahren nicht in bezug auf das Lebendgewicht irgendeine Pferdegruppe besonders bevorzugte. Obgleich VARo (7) beobachtet hat, dass die Ergebnisse je nach der Jahreszeit der Versuchsdauer etwas wechselnd sein können, derart, dass die Zugleistungen der Früjahrsvorführungen statistisch haben signifikant besser als die Versuchsergebnisse 
der übrigen Jahreszeiten sein können, haben wir in Anbetracht des Ganzen die unter verschiedenen Verhältnissen gemessenen Zugerfolge als in ihrer Vergleichbarkeit befriedigend anzusehen. Unter Berücksichtigung unserer praktischen Möglichkeiten für das Veranstalten von Versuchsgelegenheiten ist es offenbar, dass das Entwickeln des Zugversuchs am besten von der Grundlage des gegenwärtigen Systems aus zu entscheiden ist.

Die Forschungen von Vainikainen (4) und Lonka (1) über die Erblichkeit der Zugkraft erweisen, dass eine Auslese zur Entwicklung der Zugkraft der Pferde unter Anwendung des hiesigen Systems möglich ist. Vainikainens Ergebnisse zeigen, dass die nach Zugpunkten berechnete Korrelation zwischen Vätern und Söhnen $r=0.07 \pm 0.04$ und die zwischen Müttern und Söhnen $r=0.10 \pm 0.04$ ausmacht. Lonka hat nach Berechnungen für kleinere Materialien als Korrelation der Stufenzahlen zwischen Vätern und vierjährigen Söhnen $r=0.06 \pm 0.07$ und zwischen Vätern und fünfjährigen Söhnen $r=0.09 \pm 0.10$ erhalten. Die angeführten Ergebnisse beider Forscher sind also bei gegenseitigem Vergleich recht übereinstimmend. Da es sich um zwischen Eltern und Söhnen bestehende Korrelationen handelt, bei denen die ihnen entsprechende genetische Korrelation 0.50 ausmacht, können als Schätzung der Heritabilität der Zugkraft doppelte Werte der angeführten Koeffizienten angesehen werden. Die Heritabilität der nach dem bei uns gebräuchlichen Versuchsverfahren gemessenen Zugkraft nimmt somit wahrscheinlich einen Zahlenwert zwischen den Grenzwerten 0.12 und 0.20 an. Als Mittelwert ausgedrückt, beliefe sie sich auf etwa 0.16 , d.h. etwa 16 Prozent der Gesamtvarianz der Zugergebnisse wäre von erblichen Faktoren abhängig. Der Anteil dieser erblichen Varianz scheint also ziemlich klein, aber in Anbetracht dessen, dass z.B. die Heritabilität der aut recht viele Messungen gegründeten Melkleistung des Beobachtungsjahres bei Kühen nicht grösser als um 0.25 ist, hat der hiesige Zugversuch mit Pferden schon jetzt als befriedigend zu gelten. Dagegen ist es klar, dass der Wert der Heritabilität der Zugkraft so gering ist, dass Anlass besteht, mit allen Mitteln danach zu streben, den Zugversuch weiterzuentwickeln und die Zuverlässigkeit der Versuchsergebnisse zu verbessern. Dazu mögen sich denn auch in Zukunft gute Möglichkeiten bieten, nachdem es gelungen sein wird, das in Vorbereitung befindliche Züchtungsprogramm für Arbeitspferde einheitlicher zu gestalten und von der auf das Entwickeln des Trabers abgesehenen Züchtung deutlicher zu trennen.

Bei der Planung, den Zugversuch für Pferde so zu entwickeln, dass er unabhängig vom Lebendgewicht für alle Pferde gleich anstrengend wäre, ist als erstes zu entscheiden, ob es angemessen ist, den Zugwiderstand für grosse Pferde direkt als prozentualen Anteil vom Lebendgewicht zu vermehren. Die von VaInikainen (4) berechneten zwischen Lebendgewicht und prozentualem Zugwiderstand bestehenden negativen Korrelationen $\mathrm{r}=-0.08 \pm 0.03$ und $\mathrm{r}=-0.26 \pm 0.04$ weisen nämlich darauf hin, dass das Vergrössern der Zugkraft dem Vergrössern des Gewichtes nicht ganz proportional ist. Um diese Frage zu klären, wurden die Korrelationen zwischen den Zugergebnissen von 1159 vierjährigen Stammbuchhengsten und ihrem Lebendgewicht wie auch ihre Regressionskoeffizienten berechnet.

Das durchschnittliche Gewicht der genannten Hengste betrug $575.9 \mathrm{~kg}$ und ihr durchschnittliches kilogrammässiges Zugergebnis $371.1 \mathrm{~kg}$. Die Korrelation zwischen 
Gewicht und kilogrammässigem Zugerfolg belief sich auf $r=0.394 \pm 0.025$, woraus zu ersehen ist, dass die Anwendung des letzteren bei der Auslese zu einer sehr starken Bevorzugung grosser Pferde führen würde. Die Streuung $(\sigma)$ des Gewichtes betrug 39.2 und die der Zugergebnisse $40.3 \mathrm{~kg}$. Als Wert des Regressionskoeffizienten ergab sich 0.40, was erweist, dass jedem Anstieg um ein Kilogramm Lebendgewicht im Mittel ein Zunehmen um 0.4 kg im Zugergebnis entspricht. Die relative Zugleistung machte bei dem betreffenden Material durchschnittlich 64.6 Prozent vom Lebendgewicht aus. Die Korrelation zwischen Gewicht und prozentualer Zugleistung betrug $\mathrm{r}=-0.161 \pm 0.029$. Der Regressionskoeffizient -0.024 erweist, dass einem Anstieg von 100 Kilogramm Lebendgewicht eine Abnahme um 2.4 Prozenteinheiten in der relativen Zugleistung entspricht. Die relative Zugfähigkeit wächst also nicht in demselben Verhältnis wie das Gewicht des Pferdes. Wenn der Zugwiderstand auf jeder Stufe als gleich grosser Prozentsatz vom Lebendgewicht bestimmt wird, geraten die grossen Pferde in eine nachteiligere Lage als die kleinen. Die Grösse des so entstehenden Belastungsfehlers kann unter Beachtung der Streuung des Lebendgewichtes zwischen den grössten und den kleinsten Pferden sogar über 5.5 Prozenteinheiten des Zugwiderstandes ausmachen. Der Unterschied der Belastung kann also schon einen Unterschied von über einer Stufe im endgültigen Zugergebnis bewirken. Daher ist es zweckmässig, die Bestimmungsgründe des Zugwiderstandes so zu präzisieren, dass der Zuganspruch jeder Stufe die verschieden schweren Pferde in eine gleich günstige Lage bringt. Dadurch vermeidet man, den kleinen Pferdetyp zu begünstigen, dessen absolute Leistungsfähigkeit jedenfalls bedeutend geringer ist als die des grossen.

Damit das Niveau der Zugergebnisse fortfahrend dasselbe wie bisher bliebe, geschähe die Präzisierung am besten`auf die Weise, dass die Zugansprüche der dem Durchschnittsgewicht vierjähriger Hengste entsprechenden Gewichtsklasse

Tabelle 1. Die gegenwärtigen und der Zugfähigkeit entsprechenden Zugwiderstände (kg) bei den verschiedenen Gewichtsklassen.

\begin{tabular}{|c|c|c|c|c|c|c|c|c|c|}
\hline \multirow[b]{3}{*}{ Stufe } & \multicolumn{7}{|c|}{ Gewichtsklasse } & \multirow{2}{*}{\multicolumn{2}{|c|}{675}} \\
\hline & \multicolumn{2}{|c|}{475} & \multicolumn{2}{|c|}{525} & \multirow[t]{2}{*}{575} & \multicolumn{2}{|c|}{625} & & \\
\hline & Gegenwärtig & $\mathrm{Neu}$ & Gegenwärtig & Neu & & Gegenwärtig & $\mathrm{Neu}$ & Gegenwärtig & $\mathrm{Neu}$ \\
\hline 0 & 95 & 106 & 105 & 111 & 115 & 125 & 118 & 135 & 119 \\
\hline 1 & 119 & 130 & 131 & 138 & 144 & 156 & 149 & 169 & 153 \\
\hline 2 & 143 & 154 & 158 & 164 & 173 & 188 & 180 & 203 & 186 \\
\hline 3 & 166 & 178 & 184 & 190 & 201 & 219 & 211 & 236 & 220 \\
\hline 4 & 190 & 201 & 210 & 216 & 230 & 250 & 243 & 270 & 254 \\
\hline 5 & 214 & 225 & 236 & 243 & 259 & 281 & 274 & 304 & 288 \\
\hline 6 & 238 & 249 & 263 & 269 & 288 & 313 & 305 & 338 & 321 \\
\hline 7 & 261 & 273 & 289 & 295 & 316 & 344 & 336 & 371 & 355 \\
\hline 8 & 285 & 296 & 315 & 321 & 345 & 375 & 368 & 405 & 389 \\
\hline 9 & 309 & 320 & 341 & 348 & 374 & 406 & 399 & 439 & 423 \\
\hline 10 & 333 & 344 & 368 & 374 & 403 & 438 & 430 & 473 & 456 \\
\hline 11 & 356 & 368 & 394 & 400 & 431 & 469 & 461 & 506 & 490 \\
\hline 12 & 380 & 391 & 420 & 426 & 460 & 500 & 493 & 540 & 524 \\
\hline
\end{tabular}


$(575 \mathrm{~kg})$ unverändert beibehalten würde. Die Gleichung der Regressionsgeraden, nach der der Zugwiderstand der übrigen Gewichtsklassen bestimmt wird, lautet

$$
\mathrm{y}=78.4-0.024 \mathrm{x},
$$

in der $\mathrm{x}$ die Gewichtsklasse bedeutet. Die Wirkung dieser Präzisierung auf die Zugwiderstände der verschieden schweren Pferde auf den verschiedenen Stufen geht aus Tabelle 1 hervor.

Die dargestellte Präzisierung des Zugwiderstandes für die Pferde verschiedenen Gewichtes ist auch damit zu begründen, dass der von der Regressionsgleichung angezeigte Anstieg der prozentualen Zugleistung bei abnehmendem Gewicht des Pferdes dem relativen Zunehmen des Bedarfs an Unterhaltfutter, von den schweren zu den leichten Gewichtsklassen übergehend, annähernd gleichläuft. Der Bedarf an Unterhaltfutter wächst je Gewichtskilogramm jedoch in einer Kurvenlinie, wie die von Olsson und Ruudvere (2, Tabelle 5 und 6) gesammelten Ergebnisse verschiedener Forscher erweisen. Die jetzt vorgeschlagene Steigerung des Zugwiderstandes für leichte Pferde bleibt daher mit Rücksicht auf den Verbrauch an Unterhaltfutter immer noch etwas zu gering, aber es lässt sich durch sie doch der gröbste Fehler bei Berechnung des Zugwiderstandes für verschieden schwere Pferde beheben.

Die erbliche Zugfähigkeit des Pferdes zu messen, ist eine recht schwierige Aufgabe. Es ist klar, dass das endgültige Zugergebnis nicht allein von den Neigungen des Pferdes, sondern auch von seiner Gewöhnung an den Zug abhängig ist. Die Bedeutung der Gewöhnung gibt wohl am besten Tabelle 2 wieder, die die Zugleistungen von wenigstens zweimal zu einem Zugversuch herangezogenen Pferden in verschiedenen Versuchen und in verschiedenem Alter darstellt. Dieses Material machen also Pferde aus, die nicht gleich bei der ersten Prüfungsgelegenheit in das Stammbuch aufgenommen worden sind. In das Material sind daher vermutlich verhältnismässig zahlreich schwache Zugtiere eingegangen, aber grösstenteils mag es doch den normalen finnischen Pferdestand vertreten, da ein schlechtes Zugergebnis nur ziemlich selten ein endgültiges Hindernis für die Aufnahme in das Stammbuch bedeutet hat. Aus der Tabelle ist zu ersehen, dass der Einfluss der Einarbeitung auf das endgültige Zugergebnis sehr gross ist, da die Ergebnisse späterer Versuche besser als die der früheren gewesen sind.

VARO (1947 a und b) hat in früheren Untersuchungen die Zugergebnisse von 25-30 Prozent wegen fehlender Übung als misslungene Zugversuche beurteilt und empfohlen, diese Ergebnisse, die einen gesonderten Verteilungsgipfel bilden, bei Beurteilung der Zugkraft von Pferden unberücksichtigt zu lassen. In Tabelle 2

Tabelle 2. Der prozentuale Zugwiderstand mehrmals geprïfter Hengste.

\begin{tabular}{|c|c|c|c|c|c|c|}
\hline \multirow{2}{*}{$\begin{array}{c}\text { Laufende } \\
\text { Versuchsnummer }\end{array}$} & \multicolumn{3}{|c|}{$\begin{array}{c}\text { Alle Hengste }(711) \\
\text { Alter } J .\end{array}$} & \multicolumn{3}{|c|}{$\begin{array}{c}\text { Wenigstens } 35 \% \text { gezogen }(587) \\
\text { Alter } J .\end{array}$} \\
\hline & 4 & 5 & $6-$ & 4 & 5 & $6-$ \\
\hline 1 & 45.9 & 50.3 & 42.3 & 58.3 & 60.9 & 55.0 \\
\hline 2 & 51.4 & 58.6 & 51.0 & 61.4 & 63.3 & 60.8 \\
\hline $3-$ & 62.5 & 52.4 & 56.4 & 62.5 & 60.5 & 62.4 \\
\hline
\end{tabular}




\begin{tabular}{lccc}
\hline & \multicolumn{3}{c}{ Anzahl der Versuche } \\
& 2 & 3 & $4-5$ \\
\hline Alle Versuche der abgelehnten Tiere & 42 & 47 & 51 \\
Die gelungenen Versuche der abgelehnten Tiere & 58 & 57 & 58 \\
Die Versuche der übrigen Tiere & 62 & 62 & 61
\end{tabular}

1) Der Zugversuch ist hier als misslungen beurteilt worden, wenn das endgültige Ergebnis höchstens $30 \%$ gewesen ist.

sind auch die durchschnittlichen Ergebnisse von Pferden dargestellt, die wenigstens $35 \%$ ihres Lebendgewichtes gezogen haben, und aus ihnen ist zu ersehen, dass sich in den Zugergebnissen, die besser als die genannte untere Grenze sind, fortgesetzte Übung offenbar viel weniger auswirkt, als die Mittelwerte aller Zugergebnisse erweisen. Von dieser Grundlage aus liesse sich denn auch die Ausserachtlassung der Zugergebnisse von 25-30\% als Folgeerscheinung eines durch mangelhafte Ưbung bewirkten Misslingens rechtfertigen. Auf der anderen Seite ist festzustellen, dass die erbliche $\mathrm{Zugfähigkeit}$ der Pferde offenbar eine auf so manchen Faktoren beruhende Neigung ist, dass sie in ihrer Gesamtheit nicht durch Messen der $\mathrm{Zugkraft}$ des Pferdes nachgewiesen werden kann. In Tabelle 3 sind die Mittelwerte dargestellt, die die endgültigen Zugergebnisse der Pferde, die wenigstens einmal nur 20-30 Prozent - oder infolge mangelhafter Übung ein misslungenes Ergebnis - erlangt haben, im Vergleich mit den übrigen zeigen. Aus den Zahlen ist zu ersehen, dass auch die geglückten Versuche der in einer Prüfung einmal zurückgebliebenen Hengste in ihrem Erfolgsniveau schwächer bleiben als die Ergebnisse derjenigen Hengste, die sogleich beim ersten Versuch, oder auch nach Versagen in späteren Proben, nur ein gelungenes Zugresultat erzielt haben. Es ist gewiss unmöglich zu sagen, ob auch auf die späteren Ergebnisse der einmal versagenden Pferde weiterhin die unzulängliche Utbung herabsetzend eingewirkt hat oder ob es sich hier um eine erbliche Neigung handelt, die unabhängig von der Zugkraft das endgültige Zugergebnis vermindert. Es kann z.B. ein Charakterfehler mitspielen, der sich am empfindlichsten als ein schon auf den Anfangsstufen eintretendes Abschliessen des Versuchs äussert, der aber auch auf höhere Stufen gelangte Hengste hemmen kann, ihre Kräfte bis aufs äusserste anzuspannen. Die praktische Züchtungsarbeit ist gewiss nur an Pferden interessiert, die die Zugprobe einwandfrei bestehen, und ihr ist es gleichgültig, auf welche Weise die Ursachen des Misslingens analysiert werden. Da aber das Misslingen des Versuchs demgemäss ausser auf dem Mangel an Übung auch auf erblichen Neigungen beruhen kann, sind die misslungenen Versuchsergebnisse bei Beurteilung der A r be i t s f ä higk e it der Pferde nicht ausser acht zu lassen. Das Bestreben sollte also dahin gehen, die Pferde schon bis zum ersten Versuch an das Ziehen zu gewöhnen, damit sie aus Mangel an Úbung nicht versagten. Auf der anderen Seite sollten die Ergebnisse auf die endgültige Beurteilung des Pferdes einwirken, da sie im Pferde 
Mängel aufdecken mögen, die seine Leistungsfähigkeit herabsetzen. Zu diesen Zielsetzungen würde man vielleicht am besten dadurch gelangen, dass der Mittelwert aller Zugergebnisse der wiederholt für das Stammbuch angebotenen Hengste das endgültige Zugergebnis bestimmt. Dies zwingt zu sorgfältiger Ausbildung der Pferde und sichert zugleich die Beachtung aller erblichen Neigungen bei Beurteilung des Nutzungswertes des Pferdes als Lastenziehers. Eine derartige Veränderung der Instruktion dürfte auch darum begründet sein, weil nach VAINIKAINEN (5) die bei dem ersten Angebot für das Stammbuch abgelehnten Hengste grösstenteils bei den späteren Gelegenheiten aus demselben Grunde wie bei dem ersten Male zurückgewiesen worden sind.

Ausser den oben besprochenen Umständen sind bei Bestimmung der Grösse des Zugwiderstandes auch die an die verschieden alten Pferde zu stellenden Forderungen zu beachten. Ist doch der Anspruch einer entsprechenden Stufe an fünfjährige und ältere Pferde um fünf Prozenteinheiten des Zugwiderstandes grösser als bei vierjährigen. VARO (6) hat in einer früheren Untersuchung festgestellt, dass die durchschnittlichen in Stufenzahlen ausgedrückten Zugergebnisse mit zunehmendem Alter sich so vermindern, wie die folgende Zahlenreihe angibt:

$\begin{array}{lll}\text { 4jährige } & 7.24 & \text { Stufen } \\ 5-" ~ & 6.30 & " \\ \left.6-^{-1}\right) " & 5.34\end{array}$

Da einer Stufe der Anstieg um fünt Prozenteinheiten im Zugwiderstand entspricht und da ausserdem die Forderung der entsprechenden Stufe für fünfjährige 5 und für sechsjährige 10 Prozenteinheiten grösser als für vierjährige gewesen ist, kann aus den Ziffern geschlossen werden, dass, als prozentuale Zugforderung ausgedrückt, die durchschnittlichen Zugforderungen an die verschieden alten Pferde annähernd gleich gewesen sind. Aus den angeführten Stufenziffern berechnet, hätten ihre Werte in der obigen Reihenfolge 56.2, 56.5 und 56.7\% ausgemacht. Die jetzt angestellten Berechnungen erweisen ebenfalls, dass die durchschnittliche prozentuale Zugleistung vier- und fünfjähriger Pferde von annähernd gleicher Grössenordnung ist. Bei 2416 vierjährigen Pferden belief sie sich nämlich auf $60.7 \%$ und bei 1902 fünfjährigen auf $62.2 \%$. Bei Beurteilung der Bedeutung des kleinen Unterschiedes von 1.5 Prozenteinheit ist ausserdem in Betracht zu ziehen, dass auch er keineswegs einzig auf der durch das Alter bewirkten Entwicklung der Zugkraft beruht, vielmehr dürfte sich in ihm hauptsächlich die im Laufe eines Jahres in der Arbeit erlangte Ưbung ausgewirkt haben. Ein vollgültiges Material zur Erforschung der Abhängigkeit zwischen Zugfähigkeit und Alter steht vorläufig nicht zur Verfügung. Sowohl die vier- als die fünfjährig geprüften Pferde sind für diesen Zweck nicht brauchbar, da sie als vierjährige abgelehnt worden und somit ausgelesenes Material sind. Offenbar gehören zu diesen gerade solche Pferde, die als vierjährige wenig in den Zug eingearbeitet worden sind, da ihr durchschnittliches Zugergebnis nur 56.4\% oder um 4.3 Prozenteinheiten schwächer als der oben angeführte Mittelwert aller vierjährigen war. Dagegen haben sie als fünfjährige nach erlangter Ưbung annähernd ebensoviel wie die fünfjährigen durchschnittlich oder $62.0 \%$ gezogen. Eine fachkundige Untersuchung wäre auszuführen mit einem Material, das ohne Auslese in verschiedenen

1) sechsjährige und ältere. 
Lebensaltern geprüft würde. Das beste Bild geben die über die Arbeitsfähigkeit verschieden alter Pferde vorläufig oben dargestellten durchschnittlichen Ergebnisse, nach denen ihre prozentualen Zugergebnisse an sich einander vergleichbar sind. Demgemäss wäre in der Erprobungsinstruktion für Hengste eine solche Veränderung vorzunehmen, dass die Zugwiderstände für Pferde jeglichen Alters gleichartig bestimmt werden. Damit das Eintragen der Pferde in das Stammbuch nicht unbegründet verzögert wird, könnte älteren Pferden als Bedingung für ihre Aufnahme in das Stammbuch eine grössere Stufenzahl als den vierjährigen vorgeschrieben werden. Eine derartige Änderung des Systems würde die Erprobungsvorschrift bedeutend vereinfachen, und z.B. bei den Beurteilungen von Nachkommen wäre die Bewertung von Nachkommengruppen viel leichter, wenn die Stufenzahlen der Pferde verschiedenen Alters miteinander zu vergleichen wären.

Die prozentualen Zugwiderstände sind trotz der Ungleichaltrigkeit der Pferde als Mass der Zugfähigkeit recht geeignet, wie aus dem Ergebnis der Varianzanalyse hervorgeht, das unter Anwendung der obengenannten Prozentsätze für ein Material von 54 wenigstens dreimal für das Stammbuch angebotenen Hengsten berechnet worden ist. Das Material ist absichtlich auf diese Weise ausgesucht worden, weil dadurch wenigstens eines der Zugergebnisse der Hengste in einem anderen Alter als die übrigen erlangt worden ist. Der Anteil der zwischen den Hengsten bestehenden Varianz an der Gesamtvarianz der Zugergebnisse betrug mit $99 \%$ Zuverlässigkeit $17.1 \%$, was auch in diesem Fall auf eine Heritabilität gleicher Grössenordnung wie die oben beschriebenen Forschungen hinweist. Bei Beurteilung des erhaltenen Ergebnisses dürfte es begründet sein, nochmals zu wiederholen, dass das mehrfach geprüfte Hengstmaterial von schwächerer Zugfähigkeit und in gewissem Masse ausgewählt sein kann, so dass die für ein derartiges Material berechnete zwischen den Hengsten bestehende Varianz kaum grösser als der Mittelwert des gesamten Pferdematerials sein kann. Wird als Schätzung der Heritabilität der Zugkraft der früher angeführte Wert 0.16 angesehen, so wird die Bedeutung der von uns angewandten Methode zur Messung der Zugfähigkeit offenbar nicht überschätzt.

Setzt man als Wert für die Heritabilität der Zugfähigkeit 0.16 an, der nach dem Obigen recht wahrscheinlich dünkt, so kann mittels der Formel von RoBERTSON und RENDEL (3)

$$
\mathrm{b}=\frac{\mathrm{n} \cdot 0.25 \mathrm{~h}^{2}}{1+(\mathrm{n}-1) 0.25 \mathrm{~h}^{2}}
$$

berechnet werden, dass bei Anwendung der Nachkommenbeurteilung die Hengste schon auf Grund des Mittelwertes von fünf ihrer Söhne ebenso zuverlässig wie nach ihrem eigenen Zugergebnis zu schätzen wären. Zur Erlangung doppelter Zuverlässigkeit (0.32) wären 12 Söhne und zur Erzielung einer dreifachen (0.48) 22 Söhne erforderlich. Aus diesen Zahlen ist zu erkennen, dass die Wertklassifizierung der älteren Hengste durch Anwendung einer systematischen Nachkommenbeurteilung beträchtlich gesichert werden könnte. Die Bedeutung der Nachkommenbeurteilung wird bedeutend gesteigert durch die Möglichkeit, die Zugergebnisse von Stutennachkommen zu verwerten, obschon die Nachkommenbeur- 
teilung bei Stutenabkömmlingen naturgemäss infolge der Abweichung des Zugversuchs gesondert anzustellen wäre. Die Zuverlässigkeit der auf die Zugergebnisse von Stuten zu gründenden Nachkommenbeurteilung ist ebenfalls für sich vorzunehmen.

$$
\text { Schlussfolgerungen }
$$

In der Untersuchung wird festgestellt, dass die Abhängigkeit des prozentualen Zugergebnisses der Hengste vom Lebendgewicht als regressionskoeffizient berechnet $\mathrm{b}=-0.024$ ausmacht. Da die Zugfähigkeit offenbar nicht genau im Verhältnis zum Gewicht zunimmt, wird vorgeschlagen, den Zugwiderstand für Pferde verschiedenen Gewichts so zu bestimmen, dass der Zugwiderstand jeder Stufe im richtigen Verhältnis zur festgestellten Zugfähigkeit der Pferde steht.

Da die Hengste, die im Zugversuch einmal versagt — höchstens $30 \%$ gezogen - haben, auch in den übrigen Versuchen schwächer als der Durchschnitt geblieben sind, scheint das so umrissene Misslingen ausser auf unzulänglicher Übung auch auf der Neigung zu unsicherem Zug beruhen zu können. Aus diesem Grunde wird vorgeschlagen, das endgültige Zugergebnis von mehrmals in Versuchen gewesenen Pferden als Mittelwert aller Proben zu berechnen.

Weil die prozentuale Zugfähigkeit bei zunehmendem Alter der Pferde unverändert zu bleiben scheint, wird vorgeschlagen, die Zugwiderstände der verschiedenen Stufen nach denselben Grundlagen wie für verschieden alte Pferde zu berechnen.

Durch Berechnung nach den prozentualen Zugergebnissen der wenigstens in drei Zugversuchen gewesenen Pferde wird die Grösse der Heritabilität der Zugfähigkeit auf Grund der innerhalb der Klasse bestehenden Korrelation auf 0.17 geschätzt.

\section{I T E R A T U R}

(1) LoNkA, T. 1946. Hevosten vetokyvyn arvostelumahdollisuuksista. Summary: The Evaluation of the Pulling Ability of Horses. Valt. maatal. koetoim. julk. 126.

(2) Olsson, N. \& Ruudvere, A. 1955. The Nutrition of the Horse. Nutrition Abstr. Rev. 25 : 1-18.

(3) Robertson, A. \& Rendel, J. M. 1950. The use of progeny testing with artificial insemination in dairy cattle. J. Genetics 50: $21-31$.

(4) Vainikainen, V. 1946. Suomalaisen hevosen jalostuksesta. Summary: Breeding the Finnish Horse. Acta agr. fenn. 62 .

(5) —— 1947. Eräitä näkökohtia hevosjalostuksestamme. Suomen Hevosjalostusliittojen edustajiston vuosikertomus v. 1947 s: $34-43$.

(6) VARO, M. 1947. Hevosen iän ja vetovoiman keskinäisestä suhteesta. Koetoiminta ja käytäntő 6.

(7) —— 1947. Suomalaisen hevosen vetovoiman kehittämisestä. Summary: The Development of the Finnish Horses Pulling Power. Maatal.tiet. aikak. 19: 69-82. 


\title{
SELOSTUS:
}

\section{ERIPAINOISILLE JA ERI-IKÄISILLE HEVOSILLE ASETETTAVA VETOVASTUS}

\author{
Mikko Varo ja Viljo Vainikainen \\ Kotieläinjalostuslaitos, Tikkurila
}

Tutkimuksessa todetaan, että oriiden prosenttisen vetotuloksen riippuvuus elopainosta regressiokertoimena laskien on $\mathrm{b}=-\mathbf{0 . 0 2 4}$. Koska vetokyky ei ilmeisesti lisäänny tarkoin painon suhteessa, ehdotetaan vetovastus määritettäväksi eri painoisille hevosille siten, että kunkin portaan vetovastus on oikeassa suhteessa hevosten todettuun vetokykyyn.

Koska vetokokeessa kerran epäonnistuneet - enintään $30 \%$ vetäneet — oriit ovat muissakin kokeissaan jääneet keskitasoa heikommiksi, näyttää näin määritelty epäonnistuminen voivan johtua paitsi harjaantumattomuudesta myös taipumuksesta epävarmaan vetoon. Siitä syystä ehdotetaan useaan kertaan kokeissa olleiden hevosten lopullinen vetotulos laskettavaksi kaikkien kokeiden keskiarvona.

Sen johdosta, että prosenttinen vetokyky näyttää hevosten iän lisääntyessã pysyvän muuttumattomana ehdotetaan eri portaiden vetovastukset laskettaviksi samoin perustein eri-ikäisille hevosille.

Vähintään kolmessa vetokokeessa olleiden hevosten prosenttisista vetotuloksista laskien arvioidaan vetokyvyn heritabiliteetin suuruudeksi luokansisäisen korrelaation perasteella 0.17. 